\title{
Vascular liver disorders: radiology at the center of patient management
}

\author{
Maxime Ronot, ${ }^{1}$ and Sudhakar K. Venkatesh ${ }^{2}$ \\ ${ }^{1}$ Department of Radiology Hôpital Beaujon, HUPNVS, Clichy, France \\ ${ }^{2}$ Department of Radiology, Mayo Clinic College of Medicine, Mayo Clinic, Rochester, MN, USA
}

Vascular disorders of the liver are uncommon; however, with increased utility of screening methods, many are discovered incidentally. Multiple diseases affect vascular structures of the liver and the involvement may range from microscopic liver sinusoids to larger vessels such as portal vein and extrahepatic veins. The type and level of vascular involvement determines the clinical presentation of the disease. Vascular disorders of the liver have some common characteristics: patients are usually young, and some of the diseases cause significant microvascular alterations that frequently lead to non-cirrhotic portal hypertension. This triggers a compensatory increase in arterial perfusion that may result in the development of focal benign regenerative lesions such as focal nodular hyperplasia. Left untreated, some of the vascular disorders may lead to high morbidity and mortality.

The literature on vascular disorders of the liver is limited to a small number of case series, with only a few of them evaluating natural history, pathophysiology, and treatment. This probably explains why these entities are frequently under-recognized and poorly understood by radiologists. As invited editors for this special issue in Abdominal Radiology, we believe that abdominal radiologists should be aware of the imaging characteristics of vascular disorders to avoid misdiagnosis and help improve patient management. We were fortunate to have contributions from recognized world imaging experts in this field. We sincerely thank all the expert contributors for providing an up-to-date overview of the imaging and interventional management of vascular disorders.

This special issue aims at filling the knowledge gap with excellent pictorial reviews and also articles by interventional radiologists. We start with a basic refresher of vascular anatomy from Mathew et al. as a prelude to understand the vascular disorders. Dr. Van Wettere et al. provide an excellent overview on diagnostic features of Budd-Chiari syndrome and this is supported by Wang et al. who describe in detail the image-guided treatment of the Budd-Chiari syndrome. Dr. Thompson et al. describe venous involvement by intrahepatic tumors and its impact on hemodynamics and imaging features with CT and MR. Dr. Rodrigues et al. describe the minimally invasive endovascular therapy for portal vein thrombus. Dr. Wells et al. give a nice pictorial account of congestive liver disease which is an emerging chronic liver disease in young adults who survive nearly fatal congenital heart disease and in adulthood develop congestive hepatopathy. An excellent article from Dr. Rajesh et al. gives a detailed description of non-cirrhotic portal hypertension, a less well understood condition in the Western population but prevalent in Asian countries. This article has also been selected for CME. We would urge readers to avail this opportunity to obtain CME points as well as learn about non-cirrhotic portal hypertension. Dr. Brancatelli et al. have simplified the approach to sinusoidal dilatation and sinusoidal obstruction syndrome. Two reviews deal with congenital vascular abnormalities - hereditary hemorrhagic telangiectasia by Buscarini et al. and congenital porto-systemic shunts by Franchi-Abella et al. Lastly the special issue also focuses on tumors with a review on angiogenic tumors by Ehman et al. and benign and malignant tumors secondary to vascular disorders by Vilgrain et al.

We believe that this informative issue should help radiologists' knowledge about vascular disorders for accurate imaging diagnosis and help guide appropriate clinical management. 